\title{
Review Article \\ Role of Striatal-Enriched Tyrosine Phosphatase in Neuronal Function
}

\author{
Marija Kamceva, ${ }^{1}$ Jessie Benedict, ${ }^{1}$ Angus C. Nairn, ${ }^{2}$ and Paul J. Lombroso ${ }^{1}$ \\ ${ }^{1}$ Child Study Center, Yale University School of Medicine, New Haven, CT 06519, USA \\ ${ }^{2}$ Department of Psychiatry, Yale University School of Medicine, New Haven, CT 06519, USA
}

Correspondence should be addressed to Paul J. Lombroso; paul.lombroso@yale.edu

Received 2 February 2016; Accepted 27 March 2016

Academic Editor: Victor Anggono

Copyright (C) 2016 Marija Kamceva et al. This is an open access article distributed under the Creative Commons Attribution License, which permits unrestricted use, distribution, and reproduction in any medium, provided the original work is properly cited.

\begin{abstract}
Striatal-enriched protein tyrosine phosphatase (STEP) is a CNS-enriched protein implicated in multiple neurologic and neuropsychiatric disorders. STEP regulates key signaling proteins required for synaptic strengthening as well as NMDA and AMPA receptor trafficking. Both high and low levels of STEP disrupt synaptic function and contribute to learning and behavioral deficits. High levels of STEP are present in human postmortem samples and animal models of Alzheimer's disease, Parkinson's disease, and schizophrenia and in animal models of fragile X syndrome. Low levels of STEP activity are present in additional disorders that include ischemia, Huntington's chorea, alcohol abuse, and stress disorders. Thus the current model of STEP is that optimal levels are required for optimal synaptic function. Here we focus on the role of STEP in Alzheimer's disease and the mechanisms by which STEP activity is increased in this illness. Both genetic lowering of STEP levels and pharmacological inhibition of STEP activity in mouse models of Alzheimer's disease reverse the biochemical and cognitive abnormalities that are present. These findings suggest that STEP is an important point for modulation of proteins required for synaptic plasticity.
\end{abstract}

\section{Introduction}

There are 107 protein tyrosine phosphatases (PTPs) in the human genome and many of these play important roles in cellular function [1]. Striatal-enriched protein tyrosine phosphatase (STEP), encoded by the PTPN5 gene, is a CNS-enriched member of the PTP family [2]. PTPs are divided into tyrosinespecific phosphatases and dual-specificity phosphatases, with tyrosine-specific phosphatases further divided into intracellular PTPs and receptor-like PTPs [3]. STEP is an intracellular PTP, expressed throughout the CNS with the exception of the cerebellum [4].

Dysfunction in a growing number of PTPs contributes to the etiology of several diseases [5-7] and, as a result, PTPs, including STEP, have emerged as attractive targets for drug discovery $[8,9]$. The current model of STEP function is that it normally opposes synaptic strengthening by dephosphorylating key synaptic substrates. Substrates include subunits of glutamate receptors $\mathrm{N}$-methyl-D-aspartate receptors (NMDARs) and $\alpha$-amino-3-hydroxy-5-methyl-4-isoxazolepropionic acid receptors (AMPARs), leading to internalization of these receptor complexes [10-13]. Thus, increased expression of STEP disrupts synaptic function and is associated with a number of neuropsychiatric disorders, such as Alzheimer's disease [14-17]. Pharmacological inhibition of STEP would be predicted to alleviate synaptic dysfunction in Alzheimer's disease, and the successful effort in this area is reviewed below.

\section{Domain Structure of Major STEP Isoforms}

Like other PTPs, STEP contains a signature consensus sequence $[\mathrm{I} / \mathrm{V}] \mathrm{HCxAGxxR}[\mathrm{S} / \mathrm{T}] \mathrm{G}$ at its $\mathrm{C}$-terminus that is required for catalytic function and an upstream kinase-interacting motif (KIM) that is involved in binding to all known substrates [18-22]. The STEP family is alternatively spliced from a single STEP gene (PTPN5) and has two major isoforms, STEP $_{61}$ and STEP $_{46}$, which are differentially expressed in brain regions and at developmental times [18, 23, 24]. STEP $_{61}$ is found in multiple brain regions that include the striatum, central nucleus of the amygdala, optic nerve, hippocampus, neocortex, spinal cord, olfactory tubercle and bulb, and lateral amygdala, while $\mathrm{STEP}_{46}$ is expressed in striatum, nucleus accumbens, amygdala, and the optic nerve $[23,25]$. 
STEP $_{61}$ is abundantly expressed at birth and throughout adulthood, while $\mathrm{STEP}_{46}$ is not expressed until postnatal day 6 and increases over the first postnatal month when it plateaus to adult levels $[24,26]$. STEP isoforms are found in both excitatory and inhibitory neurons [27], as well as in glia [25, 28].

STEP $_{61}$ contains 172 additional amino acids at its aminoterminus compared to $\mathrm{STEP}_{46}$. The region contains two hydrophobic domains that are required to target $\mathrm{STEP}_{61}$ to the endoplasmic reticulum and the postsynaptic density of dendritic spines $[23,29]$; in contrast, $\mathrm{STEP}_{46}$ is primarily cytosolic [18]. STEP ${ }_{61}$ has two polyproline-rich regions that, in addition to the KIM domain, are involved in substrate binding and contribute to substrate specificity: the first polyproline domain is necessary for binding to Fyn [30], while the second is necessary for binding of Pyk2 [21].

Two additional alternatively spliced isoforms of STEP exist: $\mathrm{STEP}_{38}$ and $\mathrm{STEP}_{20}[4,18,31,32]$. While $\mathrm{STEP}_{61}$ and $\mathrm{STEP}_{46}$ both contain the signature consensus PTP sequence, $\mathrm{STEP}_{38}$ and $\mathrm{STEP}_{20}$ do not and are catalytically inactive [31]. Although these STEP isoforms remain to be fully characterized, they both contain KIM domains, suggesting that they may serve as variants that associate with target substrates and protect them from dephosphorylation. Both of these inactive STEP isoforms contain a 10 -amino acid sequence at their carboxyl termini that is introduced during splicing and serves an unknown function.

\section{Posttranslational Regulation of STEP}

It is important to briefly review the posttranslational regulation of STEP as it informs us of potential mechanisms in disease. STEP activity is regulated by several mechanisms that include phosphorylation, dimerization, proteolytic cleavage, ubiquitination, and local translation (for more extensive review, see [33]). Two of these mechanisms of normal STEP regulation, phosphorylation and ubiquitination, are important to note when understanding STEP dysregulation in Alzheimer's disease, which is discussed below.

Phosphorylation by protein kinase A (PKA) reduces STEP activity in two ways. PKA directly phosphorylates STEP $_{61}$ and STEP $_{46}$ at a regulatory serine within their KIM domains [34], introducing steric hindrance that prevents STEP from binding to its substrates. PKA also reduces STEP activity indirectly by phosphorylating DARPP-32, a potent inhibitor of protein phosphatase 1 (PP1). PP1 normally dephosphorylates STEP at the regulatory serine residue within the KIM domain; thus, inhibition of PP1 maintains STEP phosphorylation and reduces levels of the dephosphorylated, active STEP protein [35].

\section{STEP Substrates}

4.1. Mitogen-Activated Protein Kinase (MAPK) Family. The discovery of STEP substrates was an important advance in the understanding of the possible function of STEP in regulating neuronal signaling. Two members of the MAPK family of proteins are STEP substrates, the extracellular signal-regulated kinases 1 and $2(\mathrm{ERK} 1 / 2)$ and p38 [36-39]. ERK1/2 is implicated in synaptic plasticity and memory formation via its roles in stabilizing dendritic spines, initiating local protein synthesis in dendrites and spines, and involvement in nuclear transcription [40, 41]. STEP dephosphorylates the regulatory $\mathrm{Tyr}^{204}$ or $\mathrm{Tyr}^{187}$ residues in their respective activation loops, thereby inactivating ERK1/2.

The role of STEP regulation of ERK1/2 signaling has been studied in numerous ways, including infusion of a membranepermeable TAT- (transactivator of transcription-) STEP cysteine to serine mutant [TAT-STEP (C to $\mathrm{S}$ )]. This mutant isoform is catalytically inactive, as the cysteine residue is required for substrate dephosphorylation. However, TAT-STEP (C to $S$ ) still binds to its substrates but does not release them, as dephosphorylation is required for substrate release; thus, TAT-STEP ( $\mathrm{C}$ to $\mathrm{S}$ ) inhibits downstream signaling pathways $[10,37]$. ERK1/2 is necessary for the development of synaptic strengthening and the consolidation of fear memories in the lateral amygdala (LA). Infusion of TAT-STEP (C to S) into the LA rats did not affect the acquisition of fear memories, but there was no consolidation of these memories [42]. STEP knockout (KO) mice further established a relationship between STEP and ERK1/2, as these mice have significant elevation of phospho-ERK1/2 and increased phosphorylation of the downstream targets of ERK1/2, the transcription factors CREB and Elk1 $[43,44]$. Moreover, STEP KO mice have facilitated amygdala-dependent learning (fear conditioning [45]) and facilitated hippocampal-dependent learning (Morris water maze [44]). These studies suggested that STEP normally regulates the duration of ERK1/2 signaling and also suggested the hypothesis that elevated levels of STEP might disrupt synaptic plasticity and memory formation [37].

The MAPK, p38, is also a STEP substrate but in contrast to ERK1/2 is involved in regulation of cell death pathways and NMDAR-mediated excitotoxicity [46, 47]. Excess glutamate stimulation activates extrasynaptic GluN2B-containing NMDARs, which results in phosphorylation of p38; 338 then phosphorylates target proteins involved in cell death pathways [48]. STEP normally dephosphorylates $\mathrm{Tyr}^{182}$ in the activation loop of $\mathrm{p} 38$, inactivating the protein $[13,48]$. In addition, a number of studies have used molecular, kinetic, and structural analyses to gain insights into small differences in the KIM-containing PTPs that affect their binding to ERK2 and p38 [49-52]. Notably, both ERK1/2 and p38 regulate STEP expression levels through modulation of two phosphorylation sites adjacent to the KIM domain and dephosphorylation of these sites leads to the ubiquitination and degradation of STEP, suggesting a feedback mechanism to decrease STEP expression when ERK1/2 and p38 levels are low [53].

A study by Xu and colleagues [48] shed light on how STEP might regulate both $\mathrm{p} 38$ and ERK1/2, two proteins with very different and opposing functions. The differential regulation of these kinases by STEP depended on whether synaptic or extrasynaptic NMDARs were stimulated. STEP ${ }_{61}$ is rapidly ubiquitinated and degraded following synaptic NMDAR stimulation, resulting in activation of ERK1/2 (but not p38 signaling) and activation of synaptic strengthening and neuronal survival pathways. With increased glutamate signaling, extrasynaptic NMDARs are engaged and promote activation of calpain and the cleavage of STEP 61 within the KIM domain. 
The cleavage of the substrate-binding domain results in a STEP variant $\left(\mathrm{STEP}_{33}\right)$ that is unable to bind to and inactivate its substrates. Thus, stimulation of extrasynaptic NMDARs results in cleavage of $\mathrm{STEP}_{61}$ and activation of p38 and cell death pathways. Using a peptide corresponding to the calpain cleavage site that prevents $\mathrm{STEP}_{61}$ cleavage, there was a significant protection of neurons from glutamate-mediated excitotoxicity [48].

4.2. GluN2B and GluA2. Early studies demonstrated that dopamine signaling regulates STEP activity [34]. As mentioned above, stimulation of dopamine D1 receptors leads to activation of PKA and the phosphorylation and inactivation of STEP. Stimulation of D2 receptors has the opposite effects by reducing phosphorylation of the regulatory serine residue within the KIM domain and promoting the dephosphorylation of STEP substrates [34]. Thus, the hypothesis emerged that perhaps STEP lay between dopamine signaling and glutamate signaling through the ability of dopamine to regulate STEP activity and thereby regulate the tyrosine phosphorylation and surface expression of both NMDA and AMPA receptor complexes [10, 12, 44, 54].

Glutamate is the most abundant excitatory neurotransmitter within the CNS and binds to both metabotropic and ionotropic glutamate receptors to promote numerous cell signaling pathways in neurons $[55,56]$. NMDARs are ligandgated ion channels composed of two GluN1 and two GluN2 subunits. Activation of NMDARs requires both glutamate and glycine binding to the receptor as well as postsynaptic membrane depolarization. These receptors are selectively permeable to $\mathrm{Ca}^{2+}$ ions, which activate signaling molecules needed for long-term potentiation (LTP) and long-term depression (LTD) $[57,58]$. STEP regulates the phosphorylation of the GluN2B subunit of NMDARs via two parallel pathways, the direct dephosphorylation of GluN2B $\left(\mathrm{Tyr}^{1472}\right.$ ) as well as inactivation of the nonreceptor tyrosine kinase Fyn that phosphorylates GluN2B at that site [30, 59]. When dephosphorylated by STEP, the $\mathrm{Tyr}^{1472}$ residue of GluN2B binds to clathrin adaptor proteins and promotes internalization of GluN1/GluN2B receptors [60]. Congruent with this observation, the surface expression of GluN1/GluN2B receptor complexes is increased in STEP KO mice $[14,44]$.

The effect of STEP on NMDAR function is significant. High levels of STEP decrease NMDAR excitatory postsynaptic currents (EPSCs) and prevent the occurrence of highfrequency stimulation LTP [54]. When STEP was inhibited with a functional-inhibiting STEP antibody, NMDAR EPSCs were enhanced and LTP occluded. The administration of a noncompetitive NMDAR agonist dizocilpine (MK801) and a Src family kinase inhibitory peptide prevents these effects, suggesting a role of STEP as a "tonic brake" on LTP by opposing Src family kinase-mediated enhancement of NMDARs activity [54].

As noted above, STEP is rapidly ubiquitinated and degraded after synaptic NMDAR stimulation [48], consistent with the emerging model that STEP activity must be decreased for LTP to occur. This is consistent with a recent study that found a role for STEP in the regulation of homeostatic synaptic plasticity [61]. Prolonged neuronal activity results in the upregulation of STEP that increases removal of NMDA and AMPA receptors from synaptic membranes. Prolonged neuronal inhibition had the opposite effect, leading to the hypothesis that fine-tuning of STEP activity is necessary for maintaining proper levels of these glutamate receptors at synapses.

AMPARs are also implicated in synaptic strengthening and memory consolidation. These receptors are ligand-gated ion channels composed of subunits GluA1 to GluA4. They regulate fast synaptic transmission that depolarizes postsynaptic membranes and activates NMDARs [56, 62]. AMPAR trafficking occurs in LTD and appears to be regulated by tyrosine phosphatases that include STEP $[12,63,64]$. STEP was found to regulate the Tyr dephosphorylation of the GluA2 subunit, leading to internalization of GluA1/GluA2 receptor complexes following mGluR stimulation [12].

Local translation of STEP is increased after activation of mGluRs by the agonist DHPG (S-3,5-dihydroxyphenylglycine). This results in the tyrosine dephosphorylation of the GluA2 subunit and internalization of GluA1/GluA2 receptor complexes [12]. DHPG induces the dephosphorylation of GluA2 and internalization of AMPARs, which is decreased by the substrate-trapping protein TAT-STEP (C to S). Further, STEP KO neuronal cultures do not undergo DHPG-mediated AMPAR endocytosis, which is restored with the addition of wild type TAT-STEP protein to the STEP KO cultures. These findings suggested that, following mGluR stimulation, STEP is activated to dephosphorylate GluA2 receptors, promoting their internalization. As suggested by this model, the surface expression of GluA1/GluA2-containing AMPARs is elevated in STEP KO mice [12, 44].

\section{STEP Dysregulation in Alzheimer's Disease}

The dysregulation of STEP and glutamate receptors is implicated in several neuropsychiatric disorders, including Alzheimer's disease (AD) $[65,66]$. In $\mathrm{AD}$, abnormally high levels of $\mathrm{A} \beta$ bind to and activate $\alpha 7 \mathrm{nAChRs}$ [67-70], causing calcium influx and activation of calcineurin and PP1 and the dephosphorylation of STEP at the regulatory serine site within the KIM domain [10]. The ability of STEP to bind to its target proteins is increased and STEP substrates are dephosphorylated. To confirm that $\mathrm{A} \beta$ binding to $\alpha 7 \mathrm{nAChRs}$ and activation of PP1 were leading to activation of STEP, neuronal cultures derived from $\alpha 7 \mathrm{nAChR}$ KO mice and treated with $\mathrm{A} \beta$ were used to test whether activation of STEP was prevented in the absence of $\alpha 7 \mathrm{nAChRs}$. In fact, there was only a partial reduction STEP activation, suggesting that another mechanism was involved in activating STEP in AD.

Both mouse models of AD and neuronal cultures treated with $\mathrm{A} \beta$ were examined and found to have an accumulation of active STEP [14, 71-73]. The increase in STEP was shown not to be due to transcription or translation, suggesting that perhaps the normal degradation of STEP was disrupted. One of the effects of $A \beta$ is inhibition of the proteasome [74, 75]. Since STEP is normally degraded through the ubiquitin proteasome pathway, an increase in STEP activity was found 
to be due to an $\mathrm{A} \beta$ disruption of the ubiquitin proteasome pathway. In summary, an increase in the dephosphorylation of STEP coupled with a decrease in its degradation leads to the significant increase in STEP activity in AD.

\section{Studies of STEP in Mouse Models of AD}

$\mathrm{Tg}$-2576. The Tg-2576 AD model mouse line is a transgenic mouse line that overexpresses the 695-amino acid isoform of human amyloid precursor protein (APP). APP is an integral membrane protein, proteolysis of which generates the amyloid fibrillar form of $A \beta$, the primary component in amyloid plaques in AD brains. The mutated APP present in this mouse line contains $\mathrm{Lys}^{670} \rightarrow$ Asn and $\mathrm{Met}^{671} \rightarrow$ Leu mutations [76] and these mutations in APP are found in early onset familial AD [77-79]. At 3 months of age, Tg2576 mice perform normally in cognitive tasks and $\mathrm{A} \beta$ levels are indistinguishable from control animals. However, the Tg2576 mice show cognitive impairments by 10 months of age [76]. STEP levels are normal at the earlier time points but are significantly elevated at later time points [72].

$3 x T g-A D$. The $3 \times \mathrm{T}$ g-AD transgenic mouse line possesses three separate mutations. First, the $3 \mathrm{xTg}-\mathrm{AD}$ line has the same APP mutation present in Tg-2576 mice. Second, 3xTg$\mathrm{AD}$ mice have a presenilin mutation, one of the proteins comprising the $\gamma$-secretase complex responsible for cleaving APP at the $\mathrm{C}$-terminus of the $\mathrm{A} \beta$ domain. Third, tau is mutated in the $3 \mathrm{xTg}-\mathrm{AD}$ line. Tau is a microtubule-associated protein acting to stabilize microtubules by binding to tubulin. Tau is hyperphosphorylated in $\mathrm{AD}$, which causes paired helical filaments and destabilization of microtubules. These paired helical filaments are found in neurofibrillary tangles in patients with $\mathrm{AD}[80]$.

The 3xTg-AD mouse line has several phenotypes consistent with symptoms of human $\mathrm{AD}[81,82]$. Working memory and hippocampal memory deficits are documented, as are circadian rhythm abnormalities that are often present in early stages of $\mathrm{AD}$. These behavioral and cognitive deficits are seen in combination with $\mathrm{A} \beta$ plaque aggregation and neurofibrillary tangles, which include paired helical filaments of hyperphosphorylated tau protein [81, 82]. STEP levels were again found to be normal at earlier time points when cognitive function was unaffected but were significantly elevated at time points when cognitive deficits were present. Moreover, crossing 3xTg-AD mice with STEP KO mice reversed the cognitive deficits $[14,72]$.

\section{STEP Inhibition and AD Mouse Models}

The elevation of STEP in AD as well as the finding that genetic reduction of STEP reversed cognitive deficits in an AD mouse model validated STEP as a target for drug discovery. A high throughput screen led to the discovery of an inhibitor of STEP, 8-(trifluoromethyl)-1,2,3,4,5-benzopentathiepin-6amine hydrochloride (TC-2153) [83]. Cortical neurons treated with TC-2153 exhibit significant increase in the Tyr phosphorylation of STEP substrates GluN2B, Pyk2, and
ERK1/2. Mice injected with TC-2153 also showed increased Tyr phosphorylation of STEP substrates. Phosphatase assays were performed comparing inhibition of STEP to a panel of PTPs, including two highly related PTPs, He-PTP and PTP-SL. TC-2153 was more selective towards STEP compared with these other PTPs. Furthermore, STEP is only found in the cortex, whereas the highly related He-PTP is found in the spleen and PTP-SL in the cerebellum, tissues that lack STEP. WT and STEP KO mice were injected with TC-2153 or vehicle and the Tyr phosphorylation of ERK1/2 and Tyr phosphorylation of Pyk2 were compared in various organs. Significant increases in pERK1/2 and pPyk2 were observed only in the frontal cortex and hippocampus, but not in tissues outside of the brain or the cerebellum, where other members of the PTP family dephosphorylate ERK1/2 and Pyk2 but do not appear to be inhibited by TC-2153.

To determine the mechanism by which TC-2153 inhibits STEP, glutathione (GSH) was added in in vitro assays. It decreased the activity of TC-2153 by two orders of magnitude, implying an oxidative mechanism for STEP inhibition. STEP was then incubated with TC-2153 to monitor enzyme activity. Following $24 \mathrm{~h}$ of dialysis, STEP remained inhibited, suggesting that TC-2153 led to the formation of a covalent bond, although STEP activity could be recovered following incubation with GSH or DTT.

High-resolution tandem mass spectrometry was performed to determine whether TC-2153 modified the active site cysteine of STEP. WT STEP and a STEP mutant in which the catalytic cysteine was changed to serine were compared. Analysis of the catalytic Cys ${ }^{472}$ of STEP in the absence of TC2153 revealed a disulfide bridge between Cys ${ }^{465}$ and Cys ${ }^{472}$ which was not present in the STEP (C to S) mutant. Incubation of WT STEP with TC-2153 revealed the presence of a de novo trisulfide within the $\mathrm{Cys}^{465} / \mathrm{Cys}^{472}$ bridge, which was not observed in WT STEP alone or in the mutated STEP. These results suggested that the active site cysteine is modified by TC-2153 and that sulfur(s) from the benzopentathiepin core is retained.

TC-2153 was effective in reversing cognitive deficits in both 6- and 12-month-old 3xTg-AD mice [83]. In the novel object recognition task (NOR), mice were injected with either vehicle or TC-2153 three hours prior to the training phase and tested for memory recall after 24 hours. Post hoc analysis suggested that discrimination indexes for object memory in the AD-TC group were significantly higher than those of the AD-Veh group, while TC-2153-treated WT mice did not differ from the Veh-treated WT mice. Of interest, no significant changes were found for beta amyloid or phospho-tau levels in 12-month-old 3xTg-AD mice, suggesting that inhibition of STEP activity was sufficient to reverse cognitive deficits.

The reference memory version of the Morris water maze was then conducted [83]. 3xTg-AD mice were injected daily with TC-2153, 3 hours prior to training for peak efficacy. This STEP inhibition resulted in a reversal of memory deficits on days 5 and 6 of the task in 3xTg-AD mice. To quantify memory status, the number of mouse entries in a circular zone located around the platform, or the target zone, and in the opposite quadrants was evaluated during probe trial 


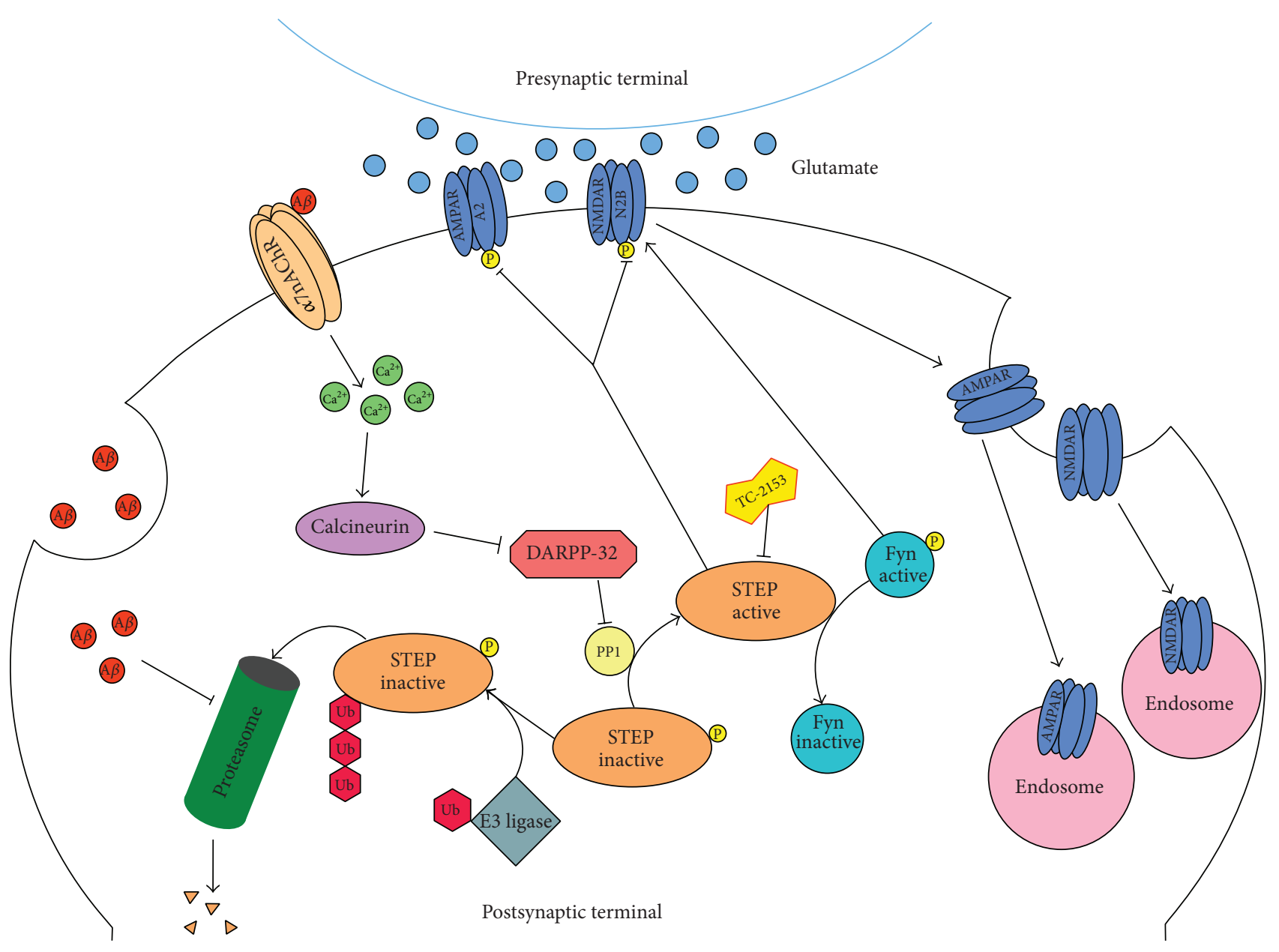

FIGURE 1: STEP signaling pathways associated with Alzheimer's disease. The binding of $\mathrm{A} \beta$ to $\alpha 7 \mathrm{nAChRs} \mathrm{results} \mathrm{in} \mathrm{activation} \mathrm{of} \mathrm{calcineurin}$ (PP2B), inhibition of DARPP-32, and activation of PP1. PP1 dephosphorylates STEP ${ }_{61}$ at a regulatory serine within the substrate-binding domain $\left(\mathrm{Ser}^{221}\right)$. Dephosphorylation of this serine residue increases the affinity of STEP for its substrates. In a parallel pathway, A $\beta$ inhibits the proteasome, thereby blocking the degradation of $\mathrm{STEP}_{61}$. Both mechanisms result in an accumulation of active $\mathrm{STEP}_{61}$. The increase in

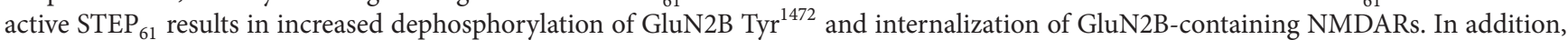
dephosphorylation of Fyn results in its inactivation. Thus, active STEP ${ }_{61}$ directly dephosphorylates GluN2B and at the same time inactivates the kinase that phosphorylates STEP $_{61}$ at Tyr $^{1472}$.

24 hours after the last acquisition day. AD mice showed no preference for the target zone, in contrast to AD mice treated with TC-2153, which spent as much time as WT mice in the target zone.

\section{Conclusion}

STEP acts by dephosphorylating regulatory tyrosine residues in substrates that include subunits of both NMDA and AMPA glutamate receptors, thereby leading to internalization of these receptor complexes (see Figure 1). Additional targets of STEP include the kinases ERK1/2, Fyn, and Pyk2 that are inactivated by dephosphorylation of regulatory tyrosines within their activation loop, thus modulating downstream signaling pathways. When STEP activity is elevated, as occurs in Alzheimer's disease, the increased internalization of glutamate receptors disrupts synaptic function and contributes to the cognitive deficits that are present. Importantly, the STEP inhibitor TC-2153 significantly improves cognitive function in $3 \mathrm{xTg}-\mathrm{AD}$ mice.

Although this review focused on Alzheimer's disease, STEP activity is elevated in several additional disorders, including Parkinson's disease [17], drug abuse [84-86], fragile $\mathrm{X}$ syndrome [16], and schizophrenia [15]. Moreover, a series of papers recently showed that low levels of STEP also disrupt synaptic function in several additional disorders, including Huntington's chorea [87, 88], cerebral ischemia [89], alcohol abuse [90-92], and stress disorders [93-95]. Thus the current model suggests that both high and low levels of STEP activity disrupt signaling pathways and contribute to neuropsychiatric and neurodegenerative disorders, making STEP an important focus of future research.

\section{Competing Interests}

The authors declare that they have no competing interests. 


\section{Authors' Contributions}

Marija Kamceva and Jessie Benedict contributed equally to this work.

\section{References}

[1] A. Alonso, J. Sasin, N. Bottini et al., "Protein tyrosine phosphatases in the human genome," Cell, vol. 117, no. 6, pp. 699-711, 2004.

[2] P. J. Lombroso, G. Murdoch, and M. Lerner, "Molecular characterization of a protein-tyrosine-phosphatase enriched in striatum," Proceedings of the National Academy of Sciences of the United States of America, vol. 88, no. 16, pp. 7242-7246, 1991.

[3] Z.-Y. Zhang, "Protein tyrosine phosphatases: structure and function, substrate specificity, and inhibitor development," Annual Review of Pharmacology and Toxicology, vol. 42, pp. 209-234, 2002.

[4] P. J. Lombroso, J. R. Naegele, E. Sharma, and M. Lerner, "A protein tyrosine phosphatase expressed within dopaminoceptive neurons of the basal ganglia and related structures," Journal of Neuroscience, vol. 13, no. 7, pp. 3054-3074, 1993.

[5] S. A. Johnson and T. Hunter, "Kinomics: methods for deciphering the kinome," Nature Methods, vol. 2, no. 1, pp. 17-25, 2005.

[6] T. Karasawa and P. J. Lombroso, "Disruption of striatal-enriched protein tyrosine phosphatase (STEP) function in neuropsychiatric disorders," Neuroscience Research, vol. 89, pp. 1-9, 2014.

[7] H. Lee, J.-S. Yi, A. Lawan, K. Min, and A. M. Bennett, "Mining the function of protein tyrosine phosphatases in health and disease," Seminars in Cell and Developmental Biology, vol. 37, pp. 66-72, 2015.

[8] A. J. Barr and S. Knapp, "MAPK-specific tyrosine phosphatases: new targets for drug discovery?" Trends in Pharmacological Sciences, vol. 27, no. 10, pp. 525-530, 2006.

[9] A. J. Barr, "Protein tyrosine phosphatases as drug targets: strategies and challenges of inhibitor development," Future Medicinal Chemistry, vol. 2, no. 10, pp. 1563-1576, 2010.

[10] E. M. Snyder, Y. Nong, C. G. Almeida et al., "Regulation of NMDA receptor trafficking by amyloid- $\beta$," Nature Neuroscience, vol. 8, no. 8, pp. 1051-1058, 2005.

[11] S. P. Braithwaite, M. Adkisson, J. Leung et al., "Regulation of NMDA receptor trafficking and function by striatal-enriched tyrosine phosphatase (STEP)," European Journal of Neuroscience, vol. 23, no. 11, pp. 2847-2856, 2006.

[12] Y. Zhang, D. V. Venkitaramani, C. M. Gladding et al., "The tyrosine phosphatase STEP mediates AMPA receptor endocytosis after metabotropic glutamate receptor stimulation," Journal of Neuroscience, vol. 28, no. 42, pp. 10561-10566, 2008.

[13] R. Poddar, I. Deb, S. Mukherjee, and S. Paul, "NR2B-NMDA receptor mediated modulation of the tyrosine phosphatase STEP regulates glutamate induced neuronal cell death," Journal of Neurochemistry, vol. 115, no. 6, pp. 1350-1362, 2010.

[14] Y. F. Zhang, P. Kurup, J. Xu et al., "Genetic reduction of the tyrosine phosphatase STEP reverses cognitive and cellular deficits in a mouse model of Alzheimer's disease," Proceedings of the National Academy of Sciences of the United States of America, vol. 107, pp. 19014-19019, 2010.

[15] N. C. Carty, J. Xu, P. Kurup et al., "The tyrosine phosphatase STEP: implications in schizophrenia and the molecular mechanism underlying antipsychotic medications," Translational Psychiatry, vol. 2, article e137, 2012.
[16] S. M. Goebel-Goody, E. D. Wilson-Wallis, S. Royston, S. M. Tagliatela, J. R. Naegele, and P. J. Lombroso, "Genetic manipulation of STEP reverses behavioral abnormalities in a fragile $\mathrm{X}$ syndrome mouse model," Genes, Brain and Behavior, vol. 11, no. 5, pp. 586-600, 2012.

[17] P. K. Kurup, J. Xu, R. A. Videira et al., "STEP ${ }_{61}$ is a substrate of the E3 ligase parkin and is upregulated in Parkinson's disease," Proceedings of the National Academy of Sciences of the United States of America, vol. 112, no. 4, pp. 1202-1207, 2015.

[18] A. Bult, F. Zhao, R. Dirkx Jr. et al., "Step61: a member of a family of brain-enriched PTPs is localized to the endoplasmic reticulum," Journal of Neuroscience, vol. 16, no. 24, pp. 7821-7831, 1996.

[19] R. Pulido, Á. Zúñiga, and A. Ullrich, "PTP-SL and STEP protein tyrosine phosphatases regulate the activation of the extracellular signal-regulated kinases ERK1 and ERK2 by association through a kinase interaction motif," The EMBO Journal, vol. 17, no. 24, pp. 7337-7350, 1998.

[20] C. Blanco-Aparicio, J. Torres, and R. Pulido, "A novel regulatory mechanism of MAP kinases activation and nuclear translocation mediated by PKA and the PTP-SL tyrosine phosphatase," The Journal of Cell Biology, vol. 147, no. 6, pp. 1129-1136, 1999.

[21] J. Xu, P. Kurup, J. A. Bartos, J. W. Hell, T. Patriarchi, and P. J. Lombroso, "Striatal-enriched protein-tyrosine phosphatase (STEP) regulates Pyk2 kinase activity," The Journal of Biological Chemistry, vol. 287, no. 25, pp. 20942-20956, 2012.

[22] J. Xu, P. Kurup, E. Foscue, and P. J. Lombroso, "Striatal-enriched protein tyrosine phosphatase regulates the PTP $\alpha /$ Fyn signaling pathway," Journal of Neurochemistry, vol. 134, no. 4, pp. 629-641, 2015.

[23] L. M. Boulanger, P. J. Lombroso, A. Raghunathan, M. J. During, P. Wahle, and J. R. Naegele, "Cellular and molecular characterization of a brain-enriched protein tyrosine phosphatase," Journal of Neuroscience, vol. 15, no. 2, pp. 1532-1544, 1995.

[24] A. Raghunathan, G. A. Matthews, P. J. Lombroso, and J. R. Naegele, "Transient compartmental expression of a family of protein tyrosine phosphatases in the developing striatum," Developmental Brain Research, vol. 91, no. 2, pp. 190-199, 1996.

[25] B. Lorber, M. Berry, W. Hendriks, J. Den Hertog, R. Pulido, and A. Logan, "Stimulated regeneration of the crushed adult rat optic nerve correlates with attenuated expression of the protein tyrosine phosphatases RPTP $\alpha$, STEP, and LAR," Molecular and Cellular Neuroscience, vol. 27, no. 4, pp. 404-416, 2004.

[26] A. Okamura, S. Goto, T. Nishi, K. Yamada, M. Yoshikawa, and Y. Ushio, "Postnatal ontogeny of striatal-enriched protein tyrosine phosphatase (STEP) in rat striatum," Experimental Neurology, vol. 145, no. 1, pp. 228-234, 1997.

[27] S. M. Goebel-Goody, K. D. Davies, R. M. Alvestad Linger, R. K. Freund, and M. D. Browning, "Phospho-regulation of synaptic and extrasynaptic N-methyl-d-aspartate receptors in adult hippocampal slices," Neuroscience, vol. 158, no. 4, pp. 1446-1459, 2009.

[28] S. Hasegawa, M. Morioka, S. Goto et al., "Expression of neuron specific phosphatase, striatal enriched phosphatase (STEP) in reactive astrocytes after transient forebrain ischemia," Glia, vol. 29, no. 4, pp. 316-329, 2000.

[29] T. Oyama, S. Goto, T. Nishi et al., "Immunocytochemical localization of the striatal enriched protein tyrosine phosphatase in the rat striatum: a light and electron microscopic study with a complementary DNA-generated polyclonal antibody," Neuroscience, vol. 69, no. 3, pp. 869-880, 1995. 
[30] T.-H. Nguyen, J. Liu, and P. J. Lombroso, "Striatal enriched phosphatase 61 dephosphorylates Fyn at phosphotyrosine 420," The Journal of Biological Chemistry, vol. 277, no. 27, pp. 2427424279, 2002.

[31] E. Sharma, F. Zhao, A. Bult, and P. J. Lombroso, "Identification of two alternatively spliced transcripts of STEP: a subfamily of brain-enriched protein tyrosine phosphatases," Molecular Brain Research, vol. 32, no. 1, pp. 87-93, 1995.

[32] A. Bult, F. Zhao, R. Dirkx Jr., A. Raghunathan, M. Solimena, and P. J. Lombroso, "STEP: a family of brain-enriched PTPs. Alternative splicing produces transmembrane, cytosolic and truncated isoforms," European Journal of Cell Biology, vol. 72, no. 4, pp. 337-344, 1997.

[33] S. M. Goebel-Goody, M. Baum, C. D. Paspalas et al., "Therapeutic implications for striatal-enriched protein tyrosine phosphatase (STEP) in neuropsychiatric disorders," Pharmacological Reviews, vol. 64, no. 1, pp. 65-87, 2012.

[34] S. Paul, G. L. Snyder, H. Yokakura, M. R. Picciotto, A. C. Nairn, and P. J. Lombroso, "The dopamine/D1 receptor mediates the phosphorylation and inactivation of the protein tyrosine phosphatase STEP via a PKA-dependent pathway," Journal of Neuroscience, vol. 20, no. 15, pp. 5630-5638, 2000.

[35] E. Valjent, V. Pascoli, P. Svenningsson et al., "Regulation of a protein phosphatase cascade allows convergent dopamine and glutamate signals to activate ERK in the striatum," Proceedings of the National Academy of Sciences of the United States of America, vol. 102, no. 2, pp. 491-496, 2005.

[36] J. J. Muñoz, C. Tárrega, C. Blanco-Aparicio, and R. Pulido, "Differential interaction of the tyrosine phosphatases PTP-SL, STEP and HePTP with the mitogen-activated protein kinases ERK1/2 and p38alpha is determined by a kinase specificity sequence and influenced by reducing agents," Biochemical Journal, vol. 372, no. 1, pp. 193-201, 2003.

[37] S. Paul, A. C. Nairn, P. Wang, and P. J. Lombroso, "NMDAmediated activation of the tyrosine phosphatase STEP regulates the duration of ERK signaling," Nature Neuroscience, vol. 6, no. 1, pp. 34-42, 2003.

[38] S. Y. Kim, H. J. Lee, Y. N. Kim et al., "Striatal-enriched protein tyrosine phosphatase regulates dopaminergic neuronal development via extracellular signal-regulated kinase signaling," Experimental Neurology, vol. 214, no. 1, pp. 69-77, 2008.

[39] S. Paul and J. A. Connor, "NR2B-NMDA receptor-mediated increases in intracellular $\mathrm{Ca}^{2+}$ concentration regulate the tyrosine phosphatase, STEP, and ERK MAP kinase signaling," Journal of Neurochemistry, vol. 114, no. 4, pp. 1107-1118, 2010.

[40] J. D. Sweatt, "Mitogen-activated protein kinases in synaptic plasticity and memory," Current Opinion in Neurobiology, vol. 14, no. 3, pp. 311-317, 2004.

[41] S. Davis, P. Vanhoutte, C. Pagès, J. Caboche, and S. Laroche, "The MAPK/ERK cascade targets both Elk-1 and cAMP response element- binding protein to control long-term potentiation-dependent gene expression in the dentate gyrus in vivo," Journal of Neuroscience, vol. 20, no. 12, pp. 4563-4572, 2000.

[42] S. Paul, P. Olausson, D. Venkitaramani et al., "The protein tyrosine phosphatase STEP gates long-term potentiation and fear memory in the lateral amygdala," Biological Psychiatry, vol. 61, no. 9, pp. 1049-1061, 2007.

[43] D. V. Venkitaramani, S. Paul, Y. Zhang et al., "Knockout of STriatal enriched protein tyrosine phosphatase in mice results in increased ERK1/2 phosphorylation," Synapse, vol. 63, no. 1 , pp. 69-81, 2009.
[44] D. V. Venkitaramani, P. J. Moura, M. R. Picciotto, and P. J. Lombroso, "Striatal-enriched protein tyrosine phosphatase (STEP) knockout mice have enhanced hippocampal memory," European Journal of Neuroscience, vol. 33, no. 12, pp. 2288-2298, 2011.

[45] P. Olausson, D. V. Venkitaramani, S. Goebel-Goody et al., "Enhanced amygdala-dependent memory formation and neuroplasticity after deletion of the tyrosine phosphatase STEP," Neuroscience, vol. 225, pp. 1-8, 2012.

[46] M. M. Semenova, A. M. J. Mäki-Hokkonen, J. Cao et al., "Rho mediates calcium-dependent activation of $\mathrm{p} 38 \alpha$ and subsequent excitotoxic cell death," Nature Neuroscience, vol. 10, no. 4, pp. 436-443, 2007.

[47] A. Ivanov, C. Pellegrino, S. Rama et al., "Opposing role of synaptic and extrasynaptic NMDA receptors in regulation of the extracellular signal-regulated kinases (ERK) activity in cultured rat hippocampal neurons," Journal of Physiology, vol. 572, no. 3, pp. 789-798, 2006.

[48] J. Xu, P. Kurup, Y. Zhang et al., "Extrasynaptic NMDA receptors couple preferentially to excitotoxicity via calpain-mediated cleavage of STEP," Journal of Neuroscience, vol. 29, no. 29, pp. 9330-9343, 2009.

[49] D. M. Francis, G. S. Kumar, D. Koveal, A. Tortajada, R. Page, and W. Peti, "The differential regulation of $\mathrm{p} 38 \alpha$ by the neuronal kinase interaction motif protein tyrosine phosphatases, a detailed molecular study," Structure, vol. 21, no. 9, pp. 1612-1623, 2013.

[50] D. M. Francis, D. Koveal, A. Tortajada, R. Page, and W. Peti, "Interaction of kinase-interaction-motif protein tyrosine phosphatases with the mitogen-activated protein kinase ERK2," PLoS ONE, vol. 9, no. 3, Article ID e91934, 2014.

[51] C. Tárrega, C. Blanco-Aparicio, J. J. Muñoz, and R. Pulido, "Two clusters of residues at the docking groove of mitogenactivated protein kinases differentially mediate their functional interaction with the tyrosine phosphatases PTP-SL and STEP," The Journal of Biological Chemistry, vol. 277, no. 4, pp. 26292636, 2002.

[52] R. Li, D.-D. Xie, J.-H. Dong et al., "Molecular mechanism of ERK dephosphorylation by striatal-enriched protein tyrosine phosphatase," Journal of Neurochemistry, vol. 128, no. 2, pp. 315329,2014

[53] S. Mukherjee, R. Poddar, I. Deb, and S. Paul, "Dephosphorylation of specific sites in the kinase-specificity sequence domain leads to ubiquitin-mediated degradation of the tyrosine phosphatase STEP," Biochemical Journal, vol. 440, no. 1, pp. 115125, 2011.

[54] K. A. Pelkey, R. Askalan, S. Paul et al., "Tyrosine phosphatase STEP is a tonic brake on induction of long-term potentiation," Neuron, vol. 34, no. 1, pp. 127-138, 2002.

[55] M. L. Mayer and N. Armstrong, "Structure and function of glutamate receptor ion channels," Annual Review of Physiology, vol. 66, pp. 161-181, 2004.

[56] S. F. Traynelis, L. P. Wollmuth, C. J. McBain et al., "Glutamate receptor ion channels: structure, regulation, and function," Pharmacological Reviews, vol. 62, no. 3, pp. 405-496, 2010.

[57] S. Cull-Candy, S. Brickley, and M. Farrant, "NMDA receptor subunits: diversity, development and disease," Current Opinion in Neurobiology, vol. 11, no. 3, pp. 327-335, 2001.

[58] N. Rebola, B. N. Srikumar, and C. Mulle, "Activity-dependent synaptic plasticity of NMDA receptors," Journal of Physiology, vol. 588, no. 1, pp. 93-99, 2010. 
[59] T. Nakazawa, S. Komai, T. Tezuka et al., "Characterization of Fyn-mediated tyrosine phosphorylation sites on GluR epsilon 2 (NR2B) subunit of the N-methyl-D-aspartate receptor," The Journal of Biological Chemistry, vol. 276, no. 1, pp. 693-699, 2001.

[60] G. Lavezzari, J. McCallum, R. Lee, and K. W. Roche, "Differential binding of the AP-2 adaptor complex and PSD-95 to the C-terminus of the NMDA receptor subunit NR2B regulates surface expression," Neuropharmacology, vol. 45, no. 6, pp. 729737, 2003.

[61] S. Jang, S. Royston, J. Xu et al., "Regulation of STEP ${ }_{61}$ and tyrosine-phosphorylation of NMDA and AMPA receptors during homeostatic synaptic plasticity," Molecular Brain, vol. 8, article 55, 2015.

[62] S. D. Santos, A. L. Carvalho, M. V. Caldeira, and C. B. Duarte, "Regulation of AMPA receptors and synaptic plasticity," Neuroscience, vol. 158, no. 1, pp. 105-125, 2009.

[63] C. M. Gladding, V. J. Collett, Z. Jia, Z. I. Bashir, G. L. Collingridge, and E. Molnár, "Tyrosine dephosphorylation regulates AMPAR internalisation in mGluR-LTD," Molecular and Cellular Neuroscience, vol. 40, no. 2, pp. 267-279, 2009.

[64] X. Chen, R. Lin, L. Chang et al., "Enhancement of long-term depression by soluble amyloid $\beta$ protein in rat hippocampus is mediated by metabotropic glutamate receptor and involves activation of p38MAPK, STEP and caspase-3," Neuroscience, vol. 253, pp. 435-443, 2013.

[65] C. G. Lau and R. S. Zukin, "NMDA receptor trafficking in synaptic plasticity and neuropsychiatric disorders," Nature Reviews Neuroscience, vol. 8, no. 6, pp. 413-426, 2007.

[66] D. V. Venkitaramani, J. Chin, W. J. Netzer et al., “ $\beta$-amyloid modulation of synaptic transmission and plasticity," Journal of Neuroscience, vol. 27, no. 44, pp. 11832-11837, 2007.

[67] K. T. Dineley, M. Westerman, D. Bui, K. Bell, K. H. Ashe, and J. D. Sweatt, " $\beta$-Amyloid activates the mitogen-activated protein kinase cascade via hippocampal $\alpha 7$ nicotinic acetylcholine receptors: in vitro and in vivo mechanisms related to Alzheimer's disease," Journal of Neuroscience, vol. 21, no. 12, pp. 4125-4133, 2001.

[68] T. R. Stevens, S. R. Krueger, R. M. Fitzsimonds, and M. R. Picciotto, "Neuroprotection by nicotine in mouse primary cortical cultures involves activation of calcineurin and L-type calcium channel inactivation," Journal of Neuroscience, vol. 23, no. 31, pp. 10093-10099, 2003.

[69] J. Hardy and D. J. Selkoe, "The amyloid hypothesis of Alzheimer's disease: progress and problems on the road to therapeutics," Science, vol. 297, no. 5580, pp. 353-356, 2002.

[70] P. N. Lacor, M. C. Buniel, L. Chang et al., "Synaptic targeting by Alzheimer's-related amyloid $\beta$ oligomers," Journal of Neuroscience, vol. 24, no. 45, pp. 10191-10200, 2004.

[71] J. Chin, J. J. Palop, J. Puoliväli et al., "Fyn kinase induces synaptic and cognitive impairments in a transgenic mouse model of Alzheimer's disease," The Journal of Neuroscience, vol. 25, no. 42, pp. 9694-9703, 2005.

[72] P. Kurup, Y. Zhang, J. Xu et al., "A $\beta$-mediated NMDA receptor endocytosis in Alzheimer's disease involves ubiquitination of the tyrosine phosphatase $\mathrm{STEP}_{61}$," The Journal of Neuroscience, vol. 30, no. 17, pp. 5948-5957, 2010.

[73] L. Zhang, J.-W. Xie, J. Yang, and Y.-P. Cao, "Tyrosine phosphatase STEP61 negatively regulates amyloid $\beta$-mediated ERK/CREB signaling pathways via $\alpha 7$ nicotinic acetylcholine receptors," Journal of Neuroscience Research, vol. 91, no. 12, pp. 1581-1590, 2013.
[74] C. G. Almeida, R. H. Takahashi, and G. K. Gouras, “ $\beta$-Amyloid accumulation impairs multivesicular body sorting by inhibiting the ubiquitin-proteasome system," The Journal of Neuroscience, vol. 26, no. 16, pp. 4277-4288, 2006.

[75] B. P. Tseng, K. N. Green, J. L. Chan, M. Blurton-Jones, and F. M. LaFerla, "A $\beta$ inhibits the proteasome and enhances amyloid and tau accumulation," Neurobiology of Aging, vol. 29, no. 11, pp. 1607-1618, 2008.

[76] K. Hsiao, P. Chapman, S. Nilsen et al., "Correlative memory deficits, $\mathrm{A} \beta$ elevation, and amyloid plaques in transgenic mice," Science, vol. 274, no. 5284, pp. 99-102, 1996.

[77] S. S. Mirra, A. Heyman, D. McKeel et al., "The consortium to establish a registry for Alzheimer's disease (CERAD). Part II. Standardization of the neuropathologic assessment of Alzheimer's disease," Neurology, vol. 41, no. 4, pp. 479-486, 1991.

[78] R. D. Terry, E. Masliah, D. P. Salmon et al., "Physical basis of cognitive alterations in Alzheimer's disease: synapse loss is the major correlate of cognitive impairment," Annals of Neurology, vol. 30, no. 4, pp. 572-580, 1991.

[79] Z. S. Khachaturian, “Diagnosis of Alzheimer's disease," Archives of Neurology, vol. 45, no. 11, pp. 1097-1105, 1985.

[80] M. Goedert, C. M. Wischik, R. A. Crowther, J. E. Walker, and A. Klug, "Cloning and sequencing of the cDNA encoding a core protein of the paired helical filament of Alzheimer disease: identification as the microtubule-associated protein tau," Proceedings of the National Academy of Sciences of the United States of America, vol. 85, no. 11, pp. 4051-4055, 1988.

[81] S. Oddo, A. Caccamo, J. D. Shepherd et al., "Triple-transgenic model of Alzheimer's Disease with plaques and tangles: intracellular A $\beta$ and synaptic dysfunction," Neuron, vol. 39, no. 3, pp. 409-421, 2003.

[82] R. Sterniczuk, M. C. Antle, F. M. LaFerla, and R. H. Dyck, "Characterization of the 3xTg-AD mouse model of Alzheimer's disease: part 2. Behavioral and cognitive changes," Brain Research, vol. 1348, pp. 149-155, 2010.

[83] J. Xu, M. Chatterjee, T. D. Baguley et al., "Inhibitors of the tyrosine phosphatase STEP reverse cognitive deficits in a mouse model of Alzheimer's disease," PLoS Biology, vol. 12, no. 8, 2014.

[84] A. A. Herrold, A. L. Persons, and T. C. Napier, "Cellular distribution of AMPA receptor subunits and mGlu5 following acute and repeated administration of morphine or methamphetamine," Journal of Neurochemistry, vol. 126, no. 4, pp. 503-517, 2013.

[85] W.-L. Sun, A. Zelek-Molik, and J. F. McGinty, "Short and long access to cocaine self-administration activates tyrosine phosphatase STEP and attenuates GluN expression but differentially regulates GluA expression in the prefrontal cortex," Psychopharmacology, vol. 229, no. 4, pp. 603-613, 2013.

[86] V. Chiodi, C. Mallozzi, J. Fan Chen et al., "Cocaine-induced changes of synaptic transmission in the striatum are modulated by adenosine $\mathrm{A}_{2 A}$ receptors and involve the tyrosine phosphatase STEP," Neuropsychopharm, vol. 39, pp. 569-578, 2014.

[87] A. Saavedra, A. Giralt, L. Rué et al., "Striatal-enriched protein tyrosine phosphatase expression and activity in Huntington's disease: a STEP in the resistance to excitotoxicity," The Journal of Neuroscience, vol. 31, no. 22, pp. 8150-8162, 2011.

[88] C. M. Gladding, M. D. Sepers, J. Xu et al., "Calpain and STriatal-Enriched protein tyrosine Phosphatase (STEP) activation contribute to extrasynaptic NMDA receptor localization in a Huntington's disease mouse model," Human Molecular Genetics, vol. 21, no. 17, Article ID dds154, pp. 3739-3752, 2012.

[89] I. Deb, N. Manhas, R. Poddar et al., "Neuroprotective role of STEP, a brain-enriched tyrosine phosphatase, in focal cerebral 
ischemia," The Journal of Neuroscience, vol. 33, no. 45, pp. 1781417826, 2013.

[90] T. R. Hicklin, P. H. Wu, R. A. Radcliffe et al., "Alcohol inhibition of the NMDA receptor function, long-term potentiation, and fear learning requires striatal-enriched protein tyrosine phosphatase," Proceedings of the National Academy of Sciences of the United States of America, vol. 108, no. 16, pp. 6650-6655, 2011.

[91] E. Darcq, S. Ben Hamida, S. Wu et al., "Inhibition of striatalenriched tyrosine phosphatase 61 in the dorsomedial striatum is sufficient to increased ethanol consumption," Journal of Neurochemistry, vol. 129, no. 6, pp. 1024-1034, 2014.

[92] R. Legastelois, E. Darcq, S. A. Wegner, P. J. Lombroso, and D. Ron, "Striatal-enriched protein tyrosine phosphatase controls responses to aversive stimuli: implication for ethanol drinking," PLoS ONE, vol. 10, no. 5, Article ID e0127408, 2015.

[93] C.-H. Yang, C.-C. Huang, and K.-S. Hsu, "Novelty exploration elicits a reversal of acute stress-induced modulation of hippocampal synaptic plasticity in the rat," The Journal of Physiology, vol. 577, no. 2, pp. 601-615, 2006.

[94] C.-H. Yang, C.-C. Huang, and K.-S. Hsu, "A critical role for protein tyrosine phosphatase nonreceptor type 5 in determining individual susceptibility to develop stress-related cognitive and morphological changes," Journal of Neuroscience, vol. 32, no. 22, pp. 7550-7562, 2012.

[95] J. Dabrowska, R. Hazra, J.-D. Guo et al., "Striatal-enriched protein tyrosine phosphatase-STEPs toward understanding chronic stress-induced activation of corticotrophin releasing factor neurons in the rat bed nucleus of the stria terminalis," Biological Psychiatry, vol. 74, no. 11, pp. 817-826, 2013. 

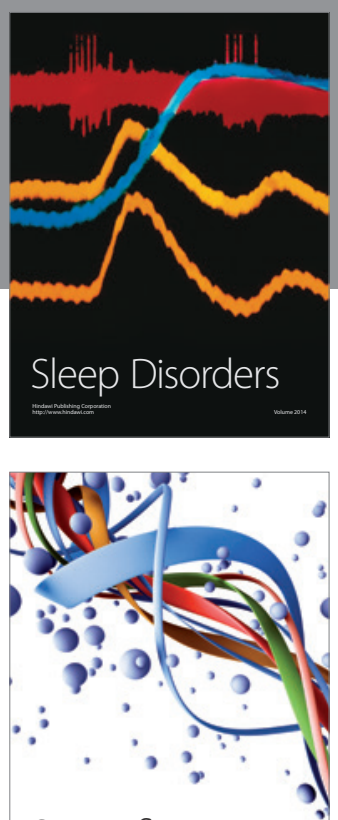

Scientifica
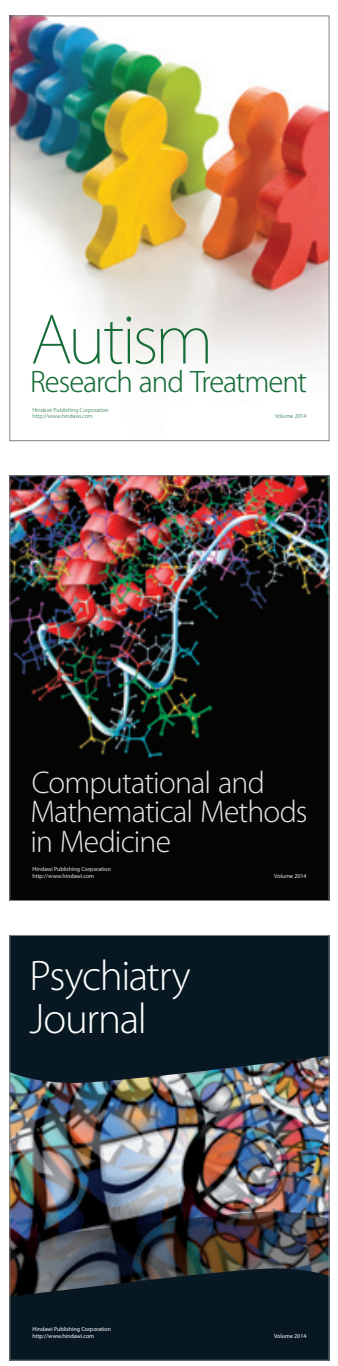
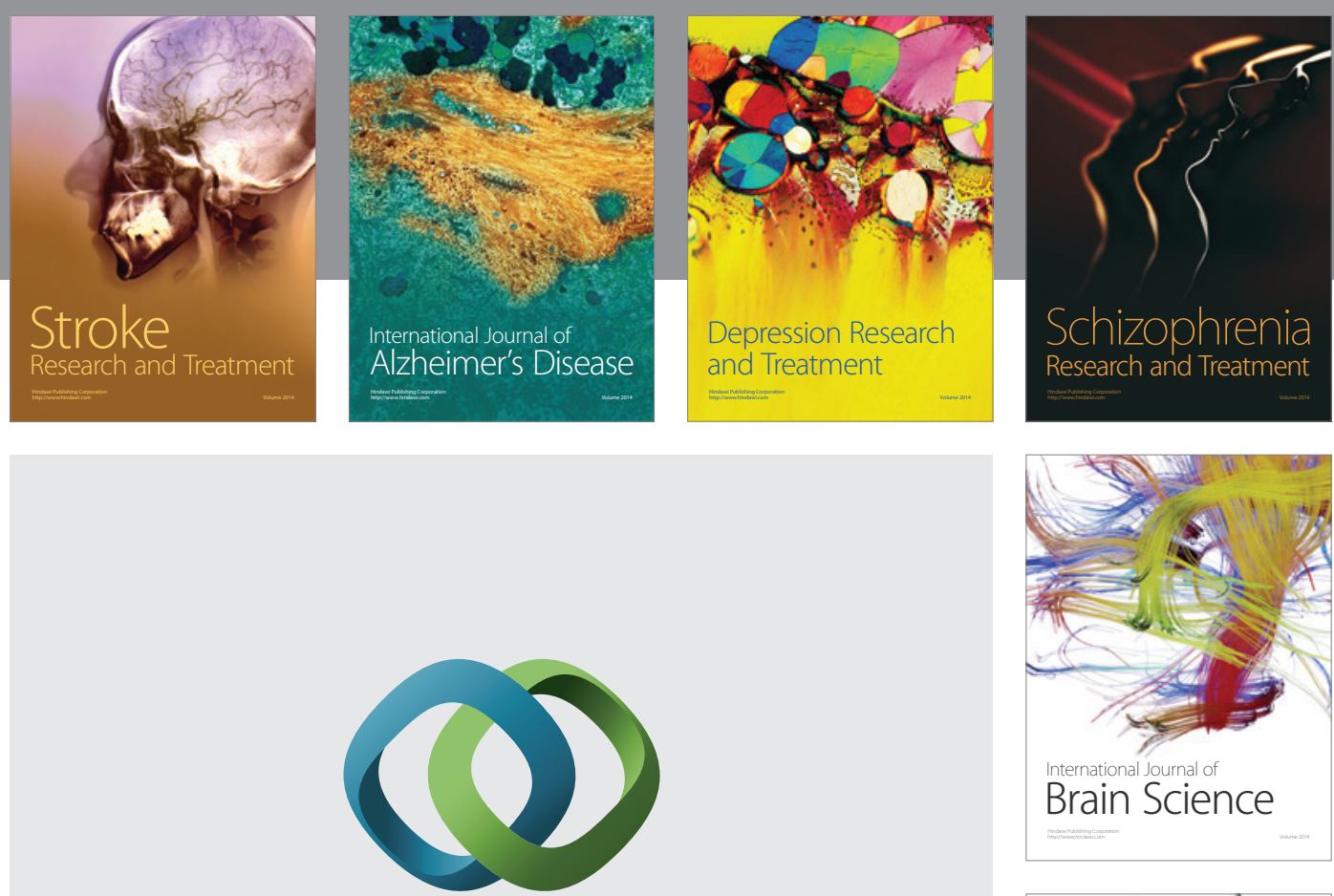

\section{Hindawi}

Submit your manuscripts at

http://www.hindawi.com
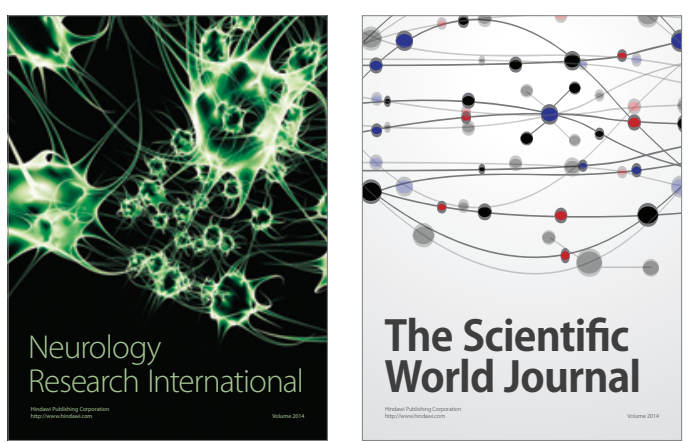

The Scientific World Journal

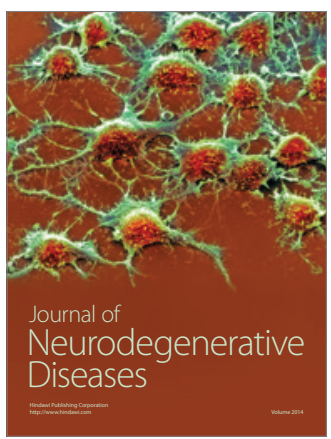

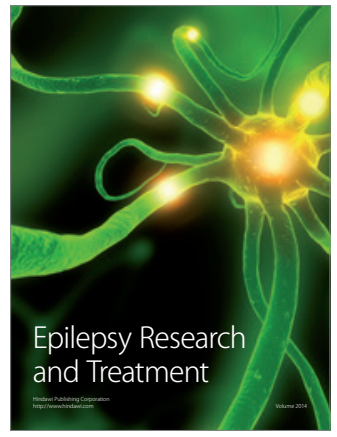

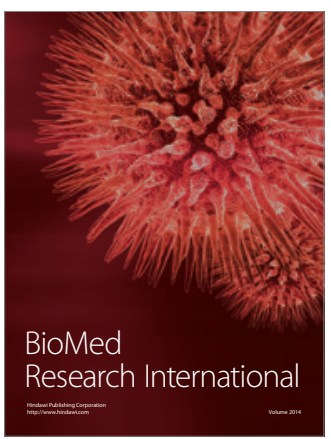

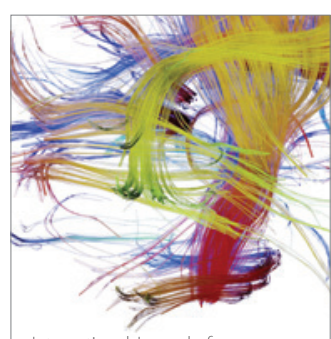

Brain Science

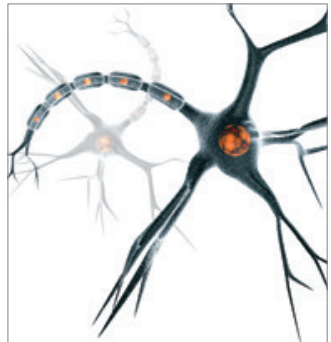

Neural Plasticity
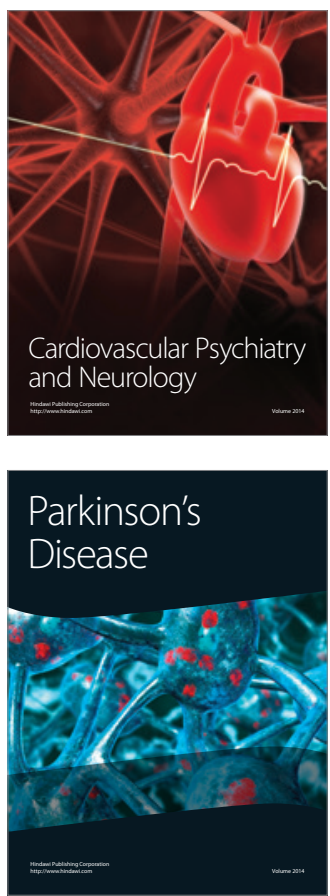